\title{
HPCGRA - An Orthogonal Designed CGRA Generator for High Performance Spatial Accelerators
}

\author{
Lucas Bragança Silva ${ }^{1}$, Michael Canesche ${ }^{1}$, \\ Ricardo Ferreira ${ }^{1}$, José Augusto M. Nacif ${ }^{1}$ \\ ${ }^{1}$ Departamento de Informática - Universidade Federal de Viçosa (UFV) \\ Avenida Peter Henry Rolfs - Minas Gerais - MG - Brazil \\ \{lucas.braganca, michael.canesche, ricardo, jnacif\}@ufv.br
}

\begin{abstract}
Recently, the increasing adoption of domain-specific architectures to execute kernels with high computing density and the exploration of sparse architectures using Systolic Arrays created the ideal scenario for using Coarsegrained reconfigurable architectures (CGRAs) to accelerate applications. Unlike Systolic Array, CGRA can run different kernel sets and keep a good balance between energy consumption and performance. In this work, we present the HPCGRA, an orthogonal designed CGRA generator for high-performance spatial accelerators. Our tool does not require any expertise in Verilog design. In our approach, the CGRA is designed and implemented in an orthogonal fashion, through wrapping the main building blocks: functional units, interconnection patterns, routing, and elastic buffer capabilities, configuration words, and memories. It optimizes and simplifies the process of creating CGRAs architectures using a portable description (JSON file) and generating a generic, scalable, and efficient Verilog RTL code with Veriloggen. The tool automatically generates CGRA with up to $46 x 66$ functional units, reaching 1.2 Tera ops/s.
\end{abstract}

\section{Introduction}

With the increasing adoption of domain-specific architectures such as Systolic Arrays to execute kernels with a high computation density, it is necessary to develop new tools to facilitate the Design Space Exploration (DSE) of the domain/application-specific architectures. Recent work has focused on generating Systolic Arrays from kernels implemented at a high level [Weng et al. 2020, Jia et al. 2020]. Other works have focused on a highperformance implementation of an essential operation such as a matrix multiplication unit by using a systolic array. Applications in Deep Learning such as deep neural networks (DNN) and convolutional neural networks $(\mathrm{CNN})$ takes profit of these high performance specialized low layer, where more than $70 \%$ of the execution time requires matrix multiplications [Asgari et al. 2019, Zhang et al. 2019]. These previous approaches focus on producing optimized architectures for only a specific set of kernels to be accelerated.

Unlike Systolic Arrays, coarse grain reconfigurable architectures (CGRAs) can run different kernel sets. However, there is a lack of tools available to help developers to generate CGRA models. The first challenge is how to describe the architecture. For instance, how to define each processing element and its basic operations? The interconnection network should provide high performance and low cost in the processing elements communications [Chin et al. 2017]. In this way, we propose a novel tool capable of generating RTL code in portable Verilog for FPGA and ASICs that automatically synthesizes 
CGRAs from a high-level description. Our approach helps the hardware developers analyze various types of architectures, including a design exploration using an orthogonal approach, to obtain data on the consumption of hardware resources, energy consumption, maximum operating frequency speed, and performance.

In this work, we present HPCGRA, an optimized tool to generate a highperformance CGRA from a single high-level description for FPGA's and ASIC's platforms. From a portable JSON configuration file, HPCGRA automatically builds an architecture. We provide orthogonal features to design, interconnect, and configure each processing element (PE). The tool also generates generic Verilog RTL code without the developer worrying about specific FPGA models or vendors. Furthermore, HPCGRA can also generate ASIC designs. Therefore, our tool contributes to reduce and to improve the design cycle. It provides a high-level abstraction layer where the developers will focus on modeling the main architecture requirements.

The contributions of our work are as follows:

- A simple method of creating generic parallel architectures from a single portable description;

- Orthogonal designed methodology;

- Portable and efficient Verilog RTL code;

- An intermediate assembly code to simplify the programming and code generation for CGRAs.

We organize this paper as follows. Section 2 provides a brief description of the proposed tool. Section 3 shows how our approach compares to related work. Section 4 evaluates our tool by generating CGRA designs. Finally, Section 5 concludes by presenting the main results and future works.

\section{HPCGRA Tool}

HPCGRA is a tool that allows the detailed description of a CGRA architecture by using a simple high-level description in JavaScript object notation (JSON) [JSON 2020]. From this description, it automatically generates all RTL code of the architecture in the Verilog language. Our generator is an open-source project based on the Veriloggen library [Takamaeda-Yamazaki 2015], which is written in Python to provide high-level facilities to produce Verilog code. Most recent related work to design specific or reconfigurable architectures is based on the language Chisel [Bachrach et al. 2012] built on top of the Scala language. We have chosen Veriloggen because Python is a more widespread language today, allowing better adhesion of the tool and possible collaborations from the community.

First, we specify a default format to perform the JSON code transformation into a CGRA architecture. It is important to highlight that our format is also orthogonal to define the main architecture features. The proposed format is possible to describe each processing element (PE) individually to design heterogeneous architectures. In the case of homogeneous ones, high-level generators could be easily created to generate our input format. This approach simplifies the elaboration of any format of the CGRA architecture. It is possible to define the set of operations for each PE. Also, we also define which are its neighbors. Section 2.1 details a simple, generic and orthogonal description format. HPCGRA also creates architectures that can be generated directly from a single command 
line, without the need for an input JSON file, where the developers only define the number of PEs with a predefined interconnection pattern set. Several CGRA architectures in the literature are based on these interconnection patterns. Table 1 depicts the main interconnection models provided by our tool. As already mentioned, this facility could be used to fast prototype homogeneous designs. However, our generator allows DSE of heterogeneous architectures.

Table 1. Interconnection patterns for the high level HPCGRA generator.

\begin{tabular}{|c|c|}
\hline Name & Description \\
\hline Mesh & Each PE communicates with its adjacent neighbors. \\
\hline One Hop & $\begin{array}{c}\text { Each PE communicates with its adjacent neighbors } \\
\text { and with its neighbors' adjacent neighbors. }\end{array}$ \\
\hline Diagonal & Same as the mesh with the addition of the diagonals neighbors. \\
\hline Hexagonal & Each PE has 6 neighbors, forming a pattern similar to a beehive. \\
\hline
\end{tabular}

\subsection{Format Specification}

In this section, we introduce the proposed JSON format to describe a CGRA architecture. We create a representation with few attributes to simplify the architecture description. The three main fields are: (1) the "shape", that represents the number of CGRA rows and columns; (2) the "data_width" field is an integer that defines the bit width of the architecture's processing data, and (3) "PEs" field is an array of objects that describes the processing elements.

In the proposed format, each PE is individually specified. This definition facilitates the construction of heterogeneous architectures in an orthogonal fashion by using wrappers. Each PE object contains the following attributes "id", "type", "neighbors", "route_type", "elastic_queue" and "isa".

The "id" attribute is the unique identifier of each PE. The "type" attribute defines whether the PE receives data from/to outside as an input and/or output node, or it only communicates with other internal PEs. The values for the "type" field are: "input", "output", and "basic", respectively. The "neighbors" attribute defines the list of IDs of the neighbors of the current PE. This approach allows the generation of any interconnection network architecture between PEs.

The attribute "route_type" determines what type of internal routing of the PE. In the current version of our tool, there are three routing options as follows:

- "no_routing": The PE has no routing capability. The function unit (FU or ALU) receives the input data from its neighbors and sends the result to any PE output ports.

- "one_routing": In addition to perform the internal operation inside the functional units, the PE can route a single signal from any neighbor PE to any/all, as shown in Figure 1.a.

- “full routing": The PE could route any neighbor' PE to any neighbor' PE as it implements an internal crossbar network, as shown in Figure 1.b. 

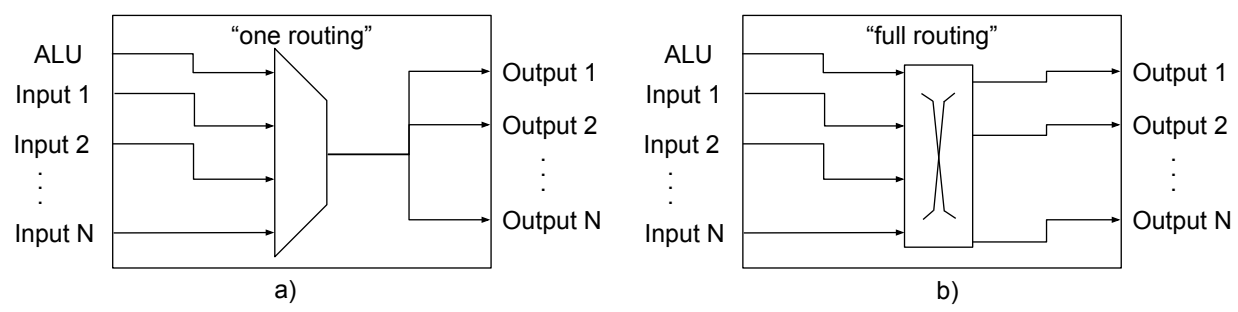

Figure 1. PE routing mechanism. a) "one_routing", b) "full_routing".

A common problem for two-dimension spatial architectures like CGRAs in pipelined design is to avoid delay mismatching after the placement and routing (P\&R) steps [Nowatzki et al. 2018]. This problem occurs when two or more paths have different arrival times when reaching a determined PE due to the P\&R decisions. This problem could be fixed by adding elastic queues at the PE entrances [Nowatzki et al. 2018]. These queues are a programmed resource where it is possible to set its size. Hence, we can create data delays and solve the delay mismatches generated by during the "P\&R" steps. The "elastic_queue" attribute specifies the maximum size of the PE queues, which can be 0 if not required.

The "isa" field corresponds to the PE instruction set. A PE performs computations with data from neighboring PEs, external input data for "input" PEs, and an internal register. In the current version of our tool, we add a set of instructions with the primary basic operations. These operations are specified by using a list inside the "isa" field. Thus, it is easy to choose which operations each PE is capable of performing. We have already implemented arithmetic and logical operations. The operations are labeled as "add", "sub", "mul”, "and", "or", "not", "madd", "addadd", "subsub", "addsub", "mux", "pass". We highlight some ternary operations, such as multiplication and sum (madd), a common operation in many applications such as matrix multiplications.

To better illustrate our input format used to generate a CGRA architecture, we present an example of a $2 \times 2$ architecture in Figure 2. This code generates a CGRA with 4 PEs and a mesh network. The PEs in the first column are input PEs, and the PEs of the last column is "output" type.

\subsection{A CGRA Instruction Set Architecture}

In this section, we present a CGRA assembly code for configuring any CGRA generated architecture. The assembly code simplifies the code generation targeting our CGRA. We use this assembly as an intermediate format. It allows applications written in different languages to compile for this format, making this tool more versatile. The language instruction fields vary according to the PE definition, which can have several neighbors and perform computation on all data from all neighbors. The basic operations showed in Section 2.1 are used in the description of the assembly instruction with the addition of the routing instruction called "route". This instruction defines which PE input will route to PE output. We use the IDs of PE to pass to the instruction. Figure 3 depicts our instruction format.

The ID fields in the format A of the instruction source operands also allow the use of the reserved word "load" or a constant numeric value. For PEs with external data entry, 


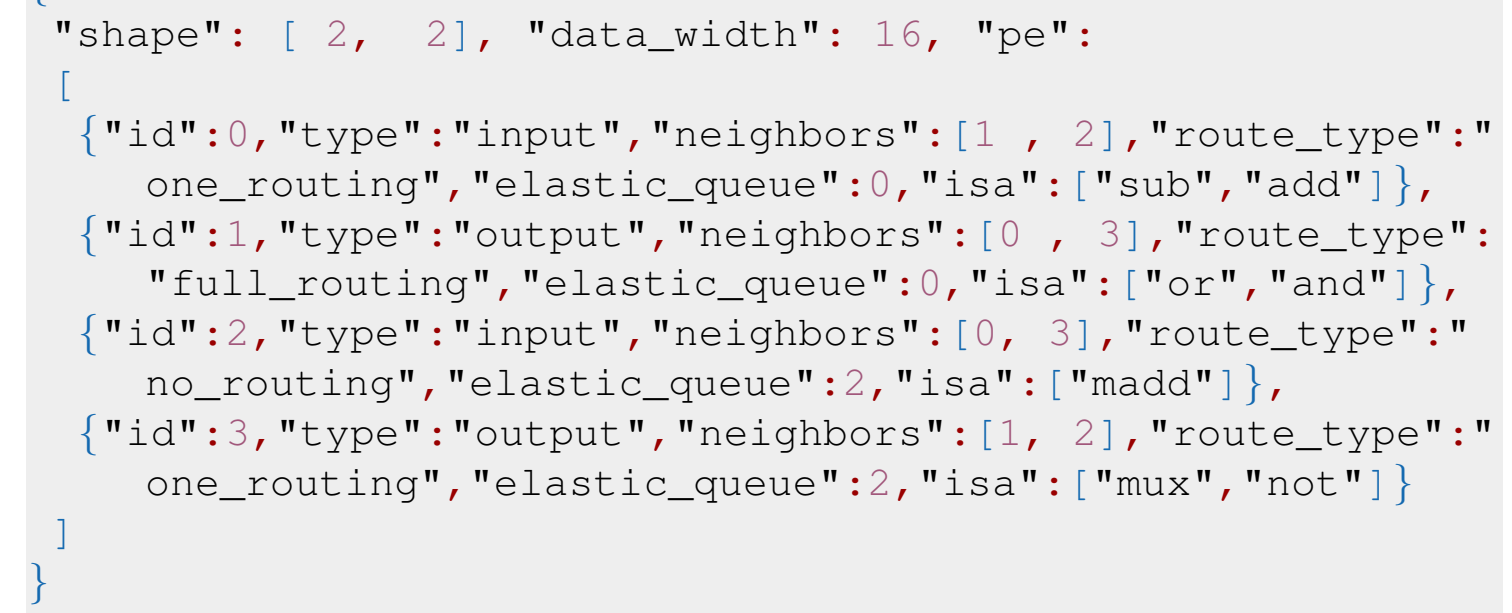

Figure 2. Sample JSON description for CGRA mesh $2 \times 2$.

Format $\mathrm{A}$ : <operation $>\$<$ pe id dst $>\#<$ delay $>\$<$ pe id src $1>\#<$ delay $>\$<$ pe id src $2>\ldots \#<$ delay $>\$<$ pe id $\operatorname{src} N>$

Format $\mathrm{B}$ : <operation=route $>\$<$ pe id src $1>\$<$ pe id src $2>$

Figure 3. Assembly Format.

we use the word "load". The numeric value operates through a constant register inside the PE, loaded at configuration time. The Format B performs an internal routing of the PE using the "route" operation, and the fields of the sources of the operand 1 and 2 are the IDs of the neighboring PEs that will be locally routed. To perform the routing of the output from the functional unit or ALU, the reserved word "alu" will place as the source field ID 1, and for output PEs, the reserved word "store" can be used in the source field 2. For PEs with an elastic queue to balance the ALU inputs, it is possible to add a delay (specified by the number of clock cycles) next to the "\#" symbol in front of the source fields.

To better illustrate what application code is like in assembly, Figure 4 presents an application for performing vector sum. In Figure 4.a the $\mathrm{C}$ code is presented, in Figure 4.b the assembly code is presented, we separate the instructions for configuring the ALU and its inputs in the first three lines and, in the other lines, the instructions for routing between the PEs. Thus, in the first instruction, we configure the ALU with the pass instruction, and its only operand is the external input port (\$load). In the second line, we make the sum in PE2 with the data of PE0 and the external input (\$load) operating and notice that a delay was added in the external input (\$load) to balance the dataflow. Finally, we configure the ALU of PE3 with the pass instruction with data provided by PE2. After configuring the ALU instructions and inputs, we carry out the routing instructions (route), where, for each PE, we need to configure from and to where your data goes. ALU's operating instructions are only one for each PE. The router instructions there may be more than one per PE. In the fourth line, the ALU exit from PE0 is routed to PE2. In the fifth line, the ALU output from PE2 is sent to PE3. Finally, in the last line, the PEU ALU output is sent to the external output (\$store) of PE3. Figure 4.c a possible mapping of the assembly code 
is shown in a $2 \times 2$ CGRA.

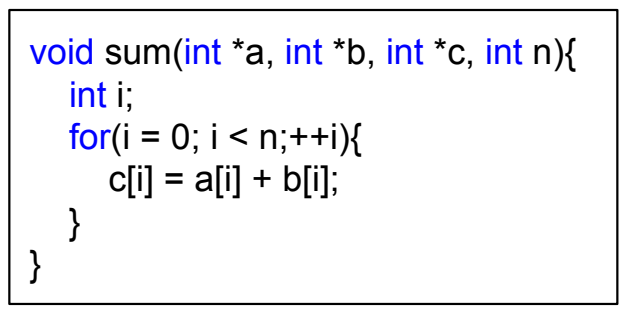

a)

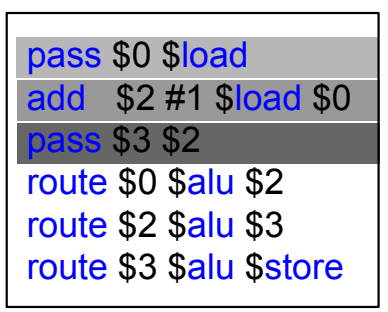

b)

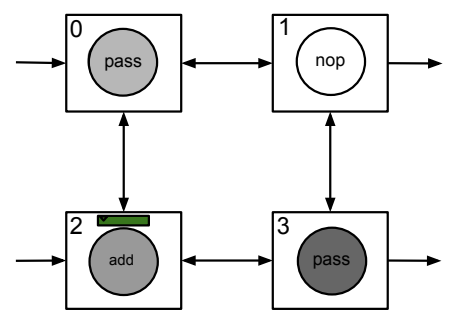

c)

Figure 4. Assembly code for vector sum.

\subsection{Orthogonal design approach}

For the generation of a CGRA architecture, we developed an orthogonal approach, where the axis of functionality, reconfiguration, and connection between the PEs are independent. In this way, architectures with different numbers of PEs perform the arrangement in the same way. For this, a PE entity is a wrapper, that the generator automatically adds the necessary resources for routing, configuration, and computation.

Figure 5 presents a representation of the dimensions of the generator. ALU (1) is the core of architecture, as it performs computations. From the ALU, other components are added, according to the architecture description. The elastic queue (2) components are connected to the ALU input ports. The routing (3) dimension is responsible for routing the PE inputs in the elastic queue and the ALU output to the PE outputs. The next dimension is interconnection (4), where each PE is connected to its neighbors according to the description. And finally, the configuration (5) dimension that is linked to all components.

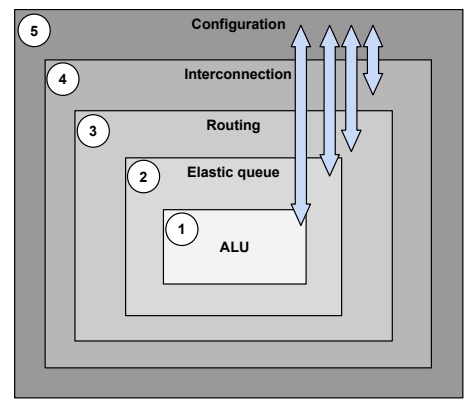

Figure 5. Representation of the dimensions of the generator.

We have developed a partial reconfiguration mechanism for each CGRA component. This mechanism allows efficiently and quickly reconfiguration. A configuration bus is automatically generated for any CGRA. It bypasses by all the PEs of the array, where rows and columns are traversed simultaneously. The bus uses registers for each $\mathrm{PE}$ throughout the route to shorten the critical path. Figure 6 shows the mechanism. It is straightforward to calculate the maximum time to configure the entire array since the configuration is sent in the pipeline at each clock cycle to the PEs of the row and column. The configuration time $\left(c t_{\text {all }}\right)$ calculation for all PEs is shown in the Equation 1, and the configuration time for a single PE $\left(c t_{p e}\right)$ in the worst case is calculated in Equation 2, 
where, Fmax is clock frequency, and $L$ is the number of rows in the array and $C$ is the number of columns.

$$
\begin{aligned}
& c t_{\text {all }}=(1 / F \max ) \times(L \times C) \\
& c t_{\text {pe }}=(1 / F \max ) \times(L+C)
\end{aligned}
$$

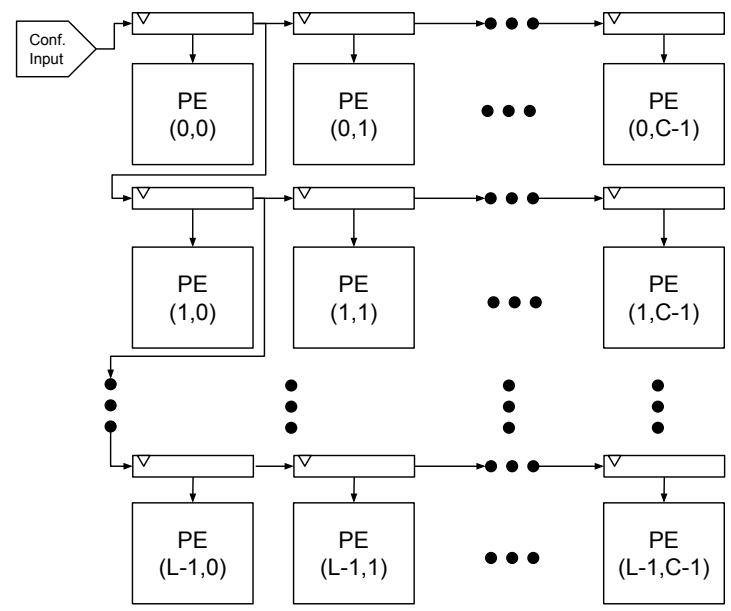

Figure 6. CGRA reconfiguration model.

\section{Related Work}

In this section, we present recent works for the generation of reconfigurable architectures. The current works have no focus on the production of generic architectures such as CGRAs. These works have focused on generating specific architectures for kernels that demand high computational density, unlike our tool that allows the user to generate a generic architecture from a JSON description.

In [Chin et al. 2018], the CGRA-ME framework is presented. Unlike our tool that uses the JSON format, CGRA-ME uses the XML format to describe CGRA architectures. For that, several standards were created, creating a specific language, with several TAGs that allow us to describe the detailed functioning of all the components of a CGRA. Our tool simplifies the description by creating components with greater granularity. Thus, the user only focuses on describing the CGRA directly.

The tool described in work [Jia et al. 2020] presents a generator of Systolic Arrays to perform vector computation. The tool uses a compiler [Genc 2020] as the frontend for the tool and uses the Chisel [Bachrach et al. 2012] infrastructure to generate the architecture's Verilog code. Unlike our tool, only architectures for specific computing are generated, making it unfeasible for systems that need to speed up different computations.

The work [Weng et al. 2020] presents a framework for DSA (design specific architecture). The DSA framework receives as input $\mathrm{C}$ kernel codes annotated with pragmas and generate a specific spatial architecture. The framework can generate an architecture that minimizes the tradeoff between performance, energy efficiency, and area. For this, the compiler has several optimization phases, such as decouple memory from computing, to leave the ideal code described in the form of a data flow graph. The work generates 
architecture through small blocks that together are capable of generating any functional architecture. These blocks are PEs, switches, memory, FIFOs, and controllers, which allow the construction of an Architecture Dataflow Graph (ADG). For a set of kernels, an ADG is iteratively optimized, adding components and removing components at random and evaluated at each iteration using an objective function. The objective function evaluates performance per square millimeter until a target value, or the graph stays stable.

\section{Evaluation}

In this section, we present the evaluations performed with the HPCGRA tool. The criterion adopted to evaluate the tool was the quality of the RTL code generated in terms of performance and resources for different types of CGRA architectures. For this, we generate CGRA with sizes 9x9, 18x18, 36x36. For each size of CGRA, we generate four types of interconnection between the PEs, the Mesh, One-hop, Diagonal, and Hexagonal model. We synthesize all architecture using the Intel/Altera infrastructure. For this, we adopted the FPGA Arria 10AX115U3F45E2SGE3 to synthesize CGRA as overlays. Arria 10 has 427200 logic blocks (ALM) and 1518 digital signal processing blocks (DSPs). We set the target clock of $400 \mathrm{MHz}$ for all synthesis because this is the maximum frequency that the FPGA Arria supports.

To validate the generator's scalability, we synthesized a heterogeneous CGRA with an array of 46x66 PEs and with 4 bits of processing word. Since the FPGA Arria 10 has only 1518 DSPs, only half of the PEs perform multiplication. CGRA used $39 \%$ of ALMs and $100 \%$ of DSP and obtained a maximum frequency of $403 \mathrm{MHz}$. We calculate this architecture's theoretical performance as follows: each PE performs an operation at each clock cycle, so the number of PEs multiplied by the cycle time in seconds is the number of operations per second that the architecture can perform. In this way, this architecture's theoretical performance is $46 * 66 * 400=1214400$ MOPs or 1.2 TOPs, showing that the generated code is scalable.

Table 2 presents a comparison of three generated CGRA sizes, where each size has three different types of internal routing. It is important to highlight that we use ADRES and HyCube columns, where the CGRAs have been generated by using the work presented in [Taras and Anderson 2019]. The ADRES architecture is compatible with our "one routing" routing model shown in Figure 1.a, where our approach uses $32 \%$ less ALMs per PE in comparison to the best result presented in the previous work [Taras and Anderson 2019]. The HyCube model is compatible with our "full routing" implementation shown in Figure 1.b, where our approach is approximately $50 \%$ more resource-efficient than [Taras and Anderson 2019]. Our generator is scalable and allows the developers to increase the number of elements, maintaining the same average resource usage per PE, as shown in Table 2.

We also perform a design exploration of several configurations by ranging the interconnection patterns, routing resources, and buffer resources. Figure 7 shows the use of resources of the Mesh and Diagonal architectures. Figures $7 \mathrm{a}, \mathrm{b}$ and $\mathrm{c}$ are of "no routing", "one routing" and "full routing" models, respectively. Each CGRA has 0, 2, and 4 sizes of elastic queues at ALU entrances. PEs with elastic queues greater than zero have higher latency in their data path, decreasing the critical path and improving the performance of the architecture against the grain and increasing the use of hardware 
Table 2. Resource usage for 16-bit CGRAs. ADRES and HyCube using FPGA STRATIX 10 [Taras and Anderson 2019] and ours using FPGA ARRIA 10. NR denotes "no_routing", OR denotes "one_routing" and "FR" denotes "full_routing".

\begin{tabular}{|c|c|c|c|c|c|c|c|c|c|c|c|}
\hline & ADRES & HyCube & $\begin{array}{c}\text { Our } \\
\text { NR }\end{array}$ & $\begin{array}{c}\text { Our } \\
\text { OR }\end{array}$ & $\begin{array}{c}\text { Our } \\
\text { FR }\end{array}$ & $\begin{array}{c}\text { Our } \\
\text { NR }\end{array}$ & $\begin{array}{c}\text { Our } \\
\text { OR }\end{array}$ & $\begin{array}{c}\text { Our } \\
\text { FR }\end{array}$ & $\begin{array}{c}\text { Our } \\
\text { NR }\end{array}$ & $\begin{array}{c}\text { Our } \\
\text { OR }\end{array}$ & $\begin{array}{c}\text { Our } \\
\text { FR }\end{array}$ \\
\hline Array Size & $4 \times 4$ & $4 \times 4$ & $9 \times 9$ & $9 \times 9$ & $9 \times 9$ & $18 \times 18$ & $18 \times 18$ & $18 \times 18$ & $36 \times 36$ & $36 \times 36$ & $36 \times 36$ \\
\hline ALM & 5051 & 5664 & 6400 & 11954 & 13827 & 26801 & 49589 & 58111 & 108709 & 202643 & 237248 \\
\hline DSP & 32 & 32 & 81 & 81 & 81 & 324 & 324 & 324 & 1296 & 1296 & 1296 \\
\hline AVG (ALM/PE) & 216 & 330 & 79 & 147 & 170 & 82 & 153 & 179 & 83 & 156 & 183 \\
\hline
\end{tabular}

resources. We can notice in the graph of Figure 7.c that the frequency increases for CGRA have elastic row sizes 2 and 4 but decreases for those that do not, with size 0 .

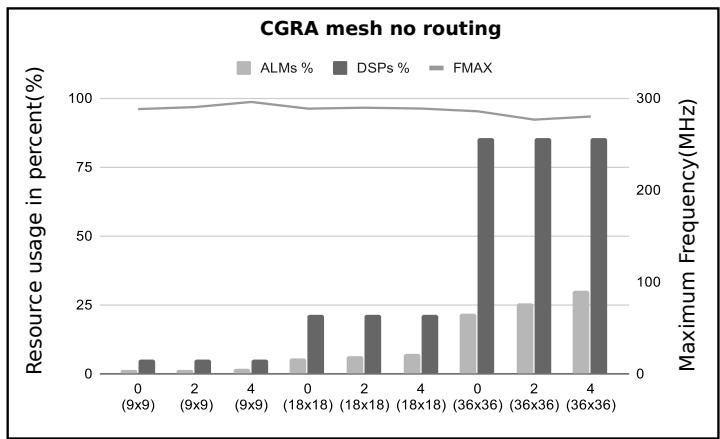

a)

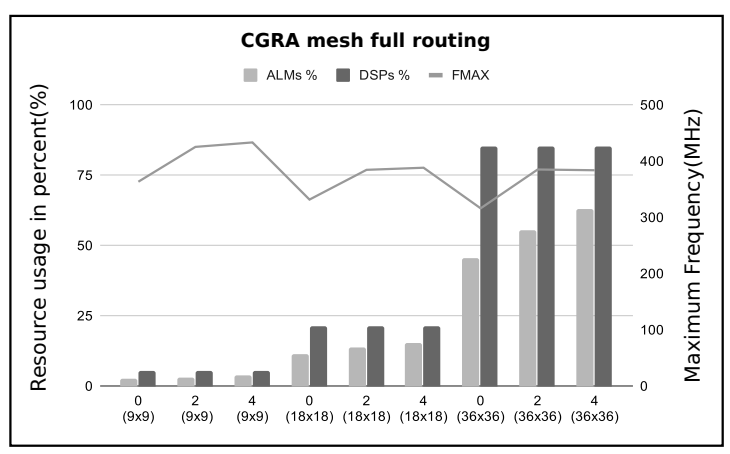

c)

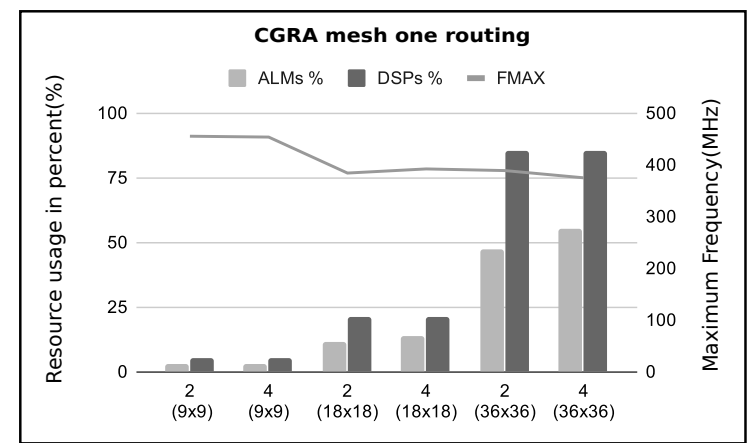

b)

Figure 7. Graph of resource usage and a maximum frequency. Graph $a, b$ and $c$ refers to the Mesh interconnection model; a) without internal routing in the $\mathrm{PE}$; b) with only one entry routing; c) with all routing possibilities.

Figure 8 shows the use of resources of the One-hop architecture. Figures $8 \mathrm{a}, \mathrm{b}$ and c are of "no routing", "one routing" and "full routing" models, respectively. Each CGRA has 0, 2, and 4 sizes of elastic queues at ALU entrances. PEs with elastic queues greater than zero have higher latency in their data path, decreasing the critical path and improving the performance of the architecture against the grain and increasing the use of hardware resources. We can notice in the graph of Figure 8.c, the frequency increases for CGRA have elastic row sizes 2 and 4 but decreases for those that do not, with size 0 . The One hop model communicates with two more neighbors than the Mesh model. It uses more hardware resources per PE, so the model with full routing Figure 8.c was able to synthesize only the sizes $9 \times 9$ and $18 \times 18$ with the elastic rows of size 0,2 , and 4 . 


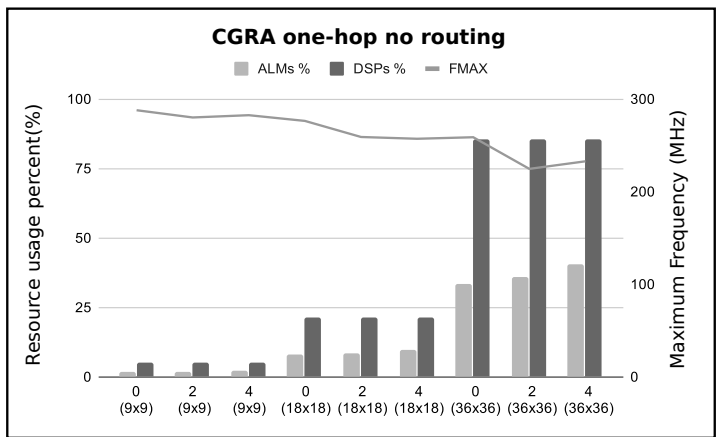

a)

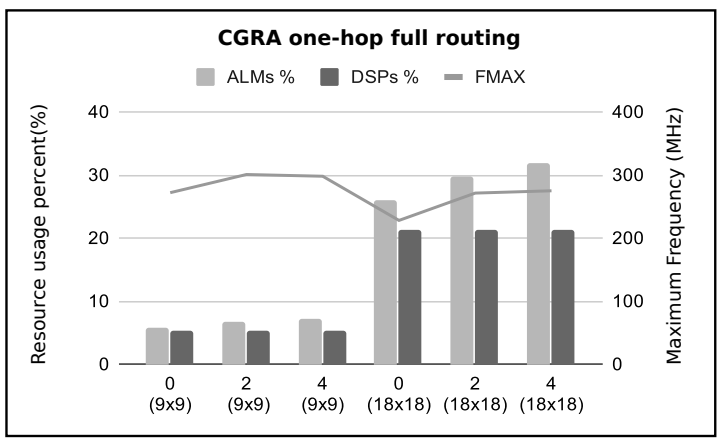

c)

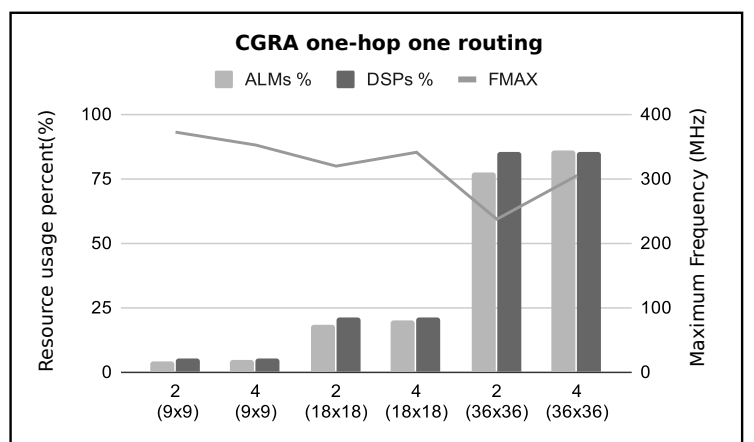

b)

Figure 8. Graph of resource usage and a maximum frequency. Graph $a, b$ and $c$ refers to the One-hop interconnection model; a) without internal routing in the PE; b) with only one entry routing; $c$ ) with all routing possibilities.

Figures $9 \mathrm{a}$ and $\mathrm{b}$ show the use of resources for the Diagonal model. In this model, each PE has eight neighbors. This model has more rout ability than the Mesh model but uses more resources. For the version "full routing," only the sizes 9x9 and 18x18 fit in the FPGA. The version without routing "no routing" showed a drop in frequency to the extent $36 \times 36$.

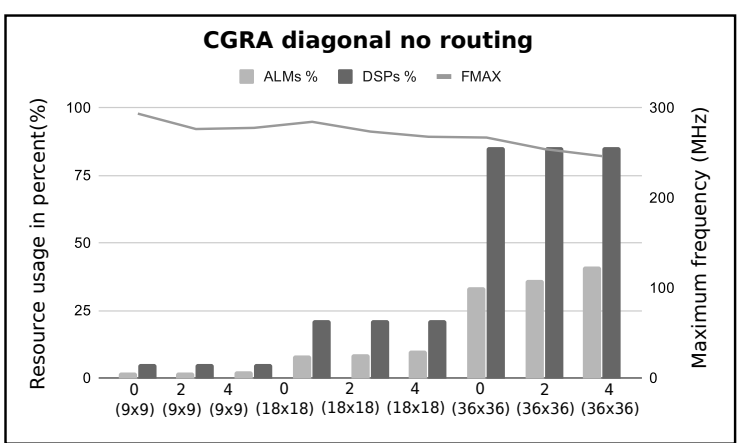

a)

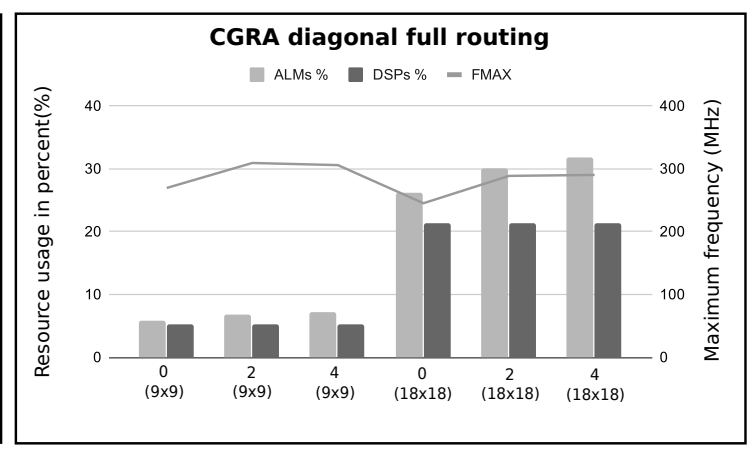

b)

Figure 9. Graph of resource usage and a maximum frequency for CGRA Diagonal interconnection model; a) without internal routing in the PE; b) with all routing possibilities.

Figures $10 \mathrm{a}$ and $\mathrm{b}$ show the results for the hexagonal architecture. In this architecture, each PE has six neighbors equal to the model of a One Hop. However, the pattern of connection between neighbors is similar to that of a hive. CGRAs with this connection 
pattern have a frequency similar to the One Hop, but with less variation when increasing the size of the matrix and the amount of latency of the elastic lines.

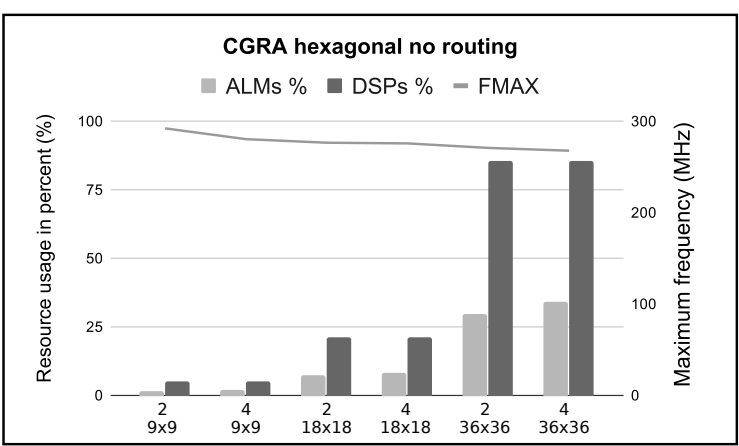

a)

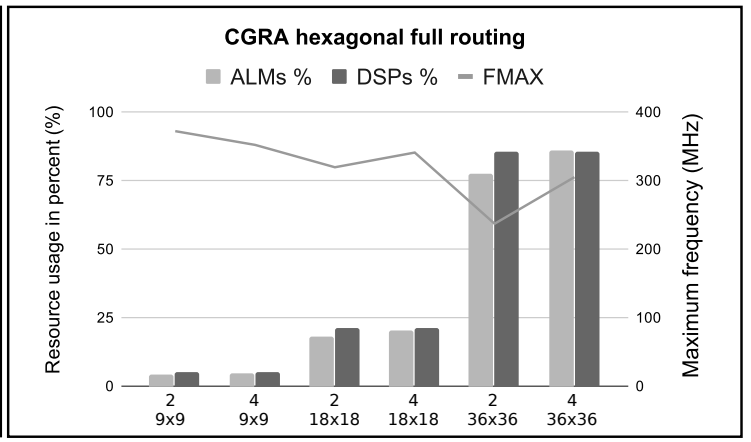

b)

Figure 10. Graph of resource usage and a maximum frequency for CGRA Hexagonal interconnection model; a) without internal routing in the PE; b) with all routing possibilities.

\section{Conclusion}

In this work, we present the HPCGRA tool. A simple way to generate Verilog code for CGRA architectures from a simple JSON description. Our tool is open source and is available on Github ${ }^{1}$. The Verilog code generated by the tool is generic and synthesizable for different FPGA platforms such as Intel/Altera or Xilinx. The synthesis results show that the frequency of the architectures is stable even with an increase in the number of PE of the CGRAs. The generator is scalable, even increasing the size of the array, the ALM/PE ratio remains stable. It was possible to synthesize architectures with 3036 PEs, with a theoretical performance of 1.2 TOPs. As future work, we will add a memory access interface through the AXI protocol facilitating the coupling of the architectures in heterogeneous high-performance computing platforms, such as Intel HARPv2 and AWS F1. Another improvement is the addition of specific functions in place of a PE ALU. For the creation of CGRA specialized in image processing algorithms, we can create a specific PE for applying filters. We also plan to design a generator for domain-specific CGRA for gene regulatory networks [Silva et al. 2017] and to integrate just-in-time compilers [Ferreira et al. 2013] to the HPCGRA tool.

\section{Acknowledgments}

This work was carried out with the support of the Coordernação de Aperfeiçoamento de Pessoal de Nível Superior - Brasil (CAPES) - Financing Code 001. Financial support from Intel Academic Compute Environment, FAPEMIG, CNPq, and Universidade Federal de Viçosa (UFV).

\section{References}

Asgari, B., Hadidi, R., Kim, H., and Yalamanchili, S. (2019). Eridanus: Efficiently running inference of dnns using systolic arrays. IEEE Micro, 39(5):46-54.

\footnotetext{
${ }^{1}$ https://github.com/lesc-ufv/hpcgra
} 
Bachrach, J., Vo, H., Richards, B., Lee, Y., Waterman, A., Avižienis, R., Wawrzynek, J., and Asanović, K. (2012). Chisel: constructing hardware in a scala embedded language. In DAC Design Automation Conference 2012, pages 1212-1221. IEEE.

Chin, S. A., Niu, K. P., Walker, M., Yin, S., Mertens, A., Lee, J., and Anderson, J. H. (2018). Architecture exploration of standard-cell and fpga-overlay cgras using the open-source cgra-me framework. In Int Symposium on Physical Design.

Chin, S. A., Sakamoto, N., Rui, A., Zhao, J., Kim, J. H., Hara-Azumi, Y., and Anderson, J. (2017). Cgra-me: A unified framework for cgra modelling and exploration. In Int Conf on Application-specific Systems, Architectures and Processors (ASAP).

Ferreira, R., Duarte, V., Meireles, W., Pereira, M., Carro, L., and Wong, S. (2013). A just-in-time modulo scheduling for virtual coarse-grained reconfigurable architectures. In Embedded Computer Systems: Architectures, Modeling, and Simulation (SAMOS).

Genc, H. (2020). A dsl for systolic arrays. https://github.com/hngenc/ systolic-array. Acessado em: 2020-08-11.

Jia, L., Lu, L., Wei, X., and Liang, Y. (2020). Generating systolic array accelerators with reusable blocks. IEEE Micro, 40(4):85-92.

JSON (2020). Introducing json. https : / / www . json . org/ json-en. html. Acessado em: 2020-07-25.

Nowatzki, T., Ardalani, N., Sankaralingam, K., and Weng, J. (2018). Hybrid optimization/heuristic instruction scheduling for programmable accelerator codesign. In Proceedings of the 27th International Conference on Parallel Architectures and Compilation Techniques, pages 1-15.

Silva, L., Almeida, D., Nacif, J., Sánchez-Osorio, I., Hernández-Martínez, C. A., and Ferreira, R. (2017). Exploring the dynamics of large-scale gene regulatory networks using hardware acceleration on a heterogeneous cpu-fpga platform. In International Conference on ReConFigurable Computing and FPGAs (ReConFig).

Takamaeda-Yamazaki, S. (2015). Pyverilog: A python-based hardware design processing toolkit for verilog hdl. In International Symposium on Applied Reconfigurable Computing, pages 451-460. Springer.

Taras, I. and Anderson, J. H. (2019). Impact of fpga architecture on area and performance of cgra overlays. In 2019 IEEE 27th Annual International Symposium on FieldProgrammable Custom Computing Machines (FCCM), pages 87-95. IEEE.

Weng, J., Liu, S., Dadu, V., Wang, Z., Shah, P., and Nowatzki, T. (2020). Dsagen: Synthesizing programmable spatial accelerators. In 2020 ACM/IEEE 47th Annual International Symposium on Computer Architecture (ISCA), pages 268-281. IEEE.

Zhang, J., Zhang, W., Luo, G., Wei, X., Liang, Y., and Cong, J. (2019). Frequency improvement of systolic array-based cnns on fpgas. In 2019 IEEE International Symposium on Circuits and Systems (ISCAS), pages 1-4. IEEE. 